\title{
Nitrogen Compound
}

National Cancer Institute

\section{Source}

National Cancer Institute. Nitrogen Compound. NCI Thesaurus. Code C1916.

Org anic or inorg anic compounds that contain nitrogen as an integral part of the molecule. 